Case Report

\title{
Lobular Carcinoma of the Breast Metastatic to the Spleen and Accessory Spleen: Report of a Case
}

\author{
Gabriel M. Groisman \\ The Institute of Pathology, Hillel Yaffe Medical Center, Hadera, Israel \\ Correspondence should be addressed to Gabriel M. Groisman; groisman@hy.health.gov.il
}

Received 31 May 2016; Accepted 21 August 2016

Academic Editor: Janina Kulka

Copyright (C) 2016 Gabriel M. Groisman. This is an open access article distributed under the Creative Commons Attribution License, which permits unrestricted use, distribution, and reproduction in any medium, provided the original work is properly cited.

\begin{abstract}
Despite the fact that accessory spleen (also known as supernumerary spleen, splenunculus, or splenule) can be found in 10$30 \%$ of patients undergoing autopsies, metastatic disease occurring in this organ has been barely reported. A case of lobular breast carcinoma metastatic to the spleen and accessory spleen found incidentally at therapeutic splenectomy for severe anemia and thrombocytopenia is described. On microscopic examination both organs revealed severe fibrocongestive changes and extramedullary hematopoiesis with no obvious carcinomatous involvement. Cytokeratin 7, estrogen receptors, and GATA3 immunohistochemistry disclosed the presence of numerous metastatic breast carcinoma cells infiltrating the splenic parenchyma. This case demonstrates that metastatic carcinoma can be encountered, although rarely, in accessory spleens and that cytokeratin stain should be performed in sections of spleens and/or accessory spleens excised from cancer patients in which the presence of malignant epithelial cells is not recognized on routine sections.
\end{abstract}

\section{Introduction}

Accessory spleen is a congenital anomaly in which there is failure of fusion between a portion of the developing splenic tissue and the main body of the spleen. Accessory spleens are present in approximately $10-30 \%$ of the population and are often an incidental finding [1-4]. Metastases from solid tumors to accessory spleens are extremely rare. To our knowledge only two such cases were reported previously $[5,6]$.

Infiltrating lobular carcinoma (ILC) represents about 5$15 \%$ of breast cancer cases [7]. It is well known that the pattern of metastatic spread of ILC is different from that of invasive ductal carcinoma, as the former may involve unusual locations such as the gastrointestinal tract, uterus, ovary, peritoneum, retroperitoneum, and meninges [7-10]. In addition, as ILC is composed of bland looking cells with a discohesive nature, its metastases can be missed in histological sections being discovered only when applying cytokeratin immunohistochemistry [11, 12].

In this report, we present the case of a patient with occult metastases of lobular carcinoma of the breast in the spleen and accessory spleen.

\section{Case Presentation}

The patient was a 49-year-old woman with a history of infiltrating lobular breast cancer. Two years before, a $4.0 \mathrm{~cm}$, irregular, spiculated mass was detected on mammography. Core needle biopsies were performed and a diagnosis of infiltrating lobular carcinoma was made (Figures $1(\mathrm{a})$ and $1(\mathrm{~b})$ ). The tumor was found to be estrogen and progesterone receptors positive and HER2/neu negative and had a proliferation index of $15-25 \%$. As subsequent clinical evaluation revealed the presence of cutaneous and bone marrow metastases (T2NXM1, stage IV) it was decided not to perform surgery and treatment with tamoxifen and goserelin was started. As a result of extensive bone marrow metastatic disease, she developed anemia, thrombocytopenia, and splenomegaly. Hypersplenism, caused by the splenomegaly, worsened the anemia and thrombocytopenia. As the hemoglobin and platelets counts decreased to $3.5 \mathrm{gr} / \mathrm{dL}$ and $8,000 / \mathrm{mm}^{3}$, respectively, and abdominal ultrasound demonstrated massive splenomegaly of size $27 \mathrm{~cm}$., splenectomy was performed. At surgery, an accessory spleen was found in the splenocolic ligament and resected. One month following surgery, 


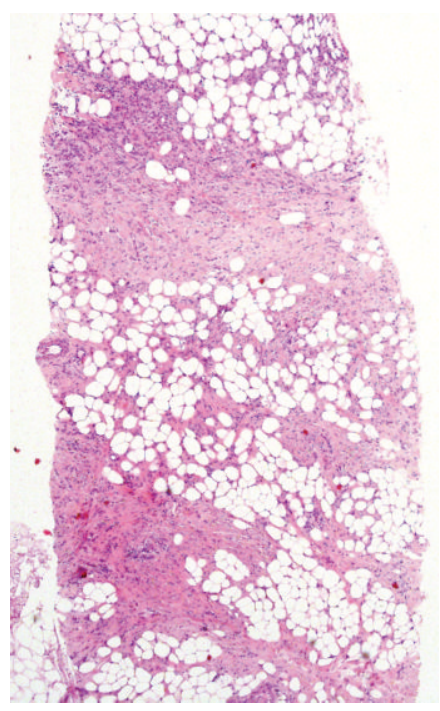

(a)

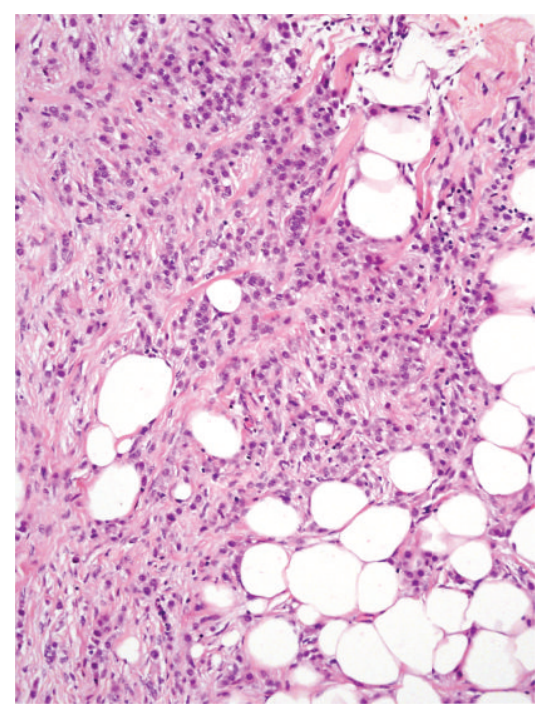

(b)

FIGURE 1: (a) Low power and (b) medium power microscopic views of the breast core biopsy showing invasive lobular carcinoma (hematoxylin and eosin stained section, magnifications $\times 40$ and $\times 200$ resp.).

the hemoglobin and platelets counts reached $9.4 \mathrm{gr} / \mathrm{dL}$ and $213,000 / \mathrm{mm}^{3}$, respectively. Seven months following the procedure, the patient feels well and is being followed up by the division of hematology/oncology.

\section{Pathological Examination}

On gross examination the spleen measured $28 \times 21 \times 12 \mathrm{~cm}$. and weighed 2,700 gr. whereas the splenunculus measured $3.0 \mathrm{~cm}$. in diameter and weighed $6 \mathrm{gr}$. The capsules of both organs were smooth, tense, and intact and the parenchyma was dark red and firm with a vague nodular pattern. Representative sections were embedded in paraffin and stained with hematoxylin and eosin. Immunohistochemistry using the streptavidin-biotin peroxidase complex method was performed on a Ventana Benchmark automatic immunostainer (Tucson, AZ, USA) with the following antibodies: cytokeratin 7 (clone OV-TL12/30, ready to use [RTU]; Dako, Glostrup, Denmark), GATA3 (clone 634913, 1:50; R\&D Systems, Minneapolis, MN, USA), estrogen receptor (clone SP1, RTU; Ventana, Tucson, AZ, USA), and E-cadherin (clone EP700Y, RTU; Cell Marque, Rocklin, CA, USA).

Histologic sections of both the spleen and the splenunculus revealed inconspicuous white pulp and marked expansion of the red pulp due to marked congestion and extensive extramedullary hematopoiesis $(\mathrm{EMH})$ distributed in a nodular pattern (Figure 2(a)). Myeloid and erythroid elements infiltrated cords and sinuses and megakaryocytes occasionally formed clusters that included forms with bizarre morphologic features (Figure 2(b)). Foci of EMH were separated by bands of reticulin and fibrous connective tissue. In addition, there was severe blood congestion, accumulation of hemosiderin-containing macrophages, and formation of fibroelastotic nodules with hemosiderin deposition (“Gamna-Gandy bodies").
The presence of metastatic carcinoma within the splenic parenchyma could not be definitely established from hematoxylin and eosin stained sections. However, small cells with occasional cytoplasmic vacuoles resembling lobular carcinoma cells were detected within the adjacent fatty tissue (Figure 2(c)). Immunohistochemically, these cells, as well as numerous additional cells widely distributed within the spleen and accessory spleen parenchymata as single units or small, loose aggregates reacted strongly with cytokeratin 7 , estrogen receptor, and GATA3 (Figures 2(d), 2(e), and 2(f)). In contrast, E-cadherin stain was negative. These results supported the diagnosis of lobular breast carcinoma metastatic to the spleen and accessory spleen.

\section{Discussion}

The occurrence of breast carcinoma metastasizing to an accessory spleen has not been reported yet. In this case, clinically unsuspected metastatic lobular breast carcinoma cells diffusely infiltrated the spleen and the accessory spleen. Microscopically, metastatic carcinoma was not clearly identified with hematoxylin and eosin stained sections although suspicious cells were present within the adjacent fatty tissue. Immunohistochemical stains, however, disclosed the presence of numerous metastatic lobular carcinoma cells within the spleen and accessory spleen parenchymata and confirmed the malignant nature of the suspicious cells observed in the adipose tissue adjacent to the spleens. These findings demonstrated that the severe splenomegaly resulted not only from severe congestion and EMH but also from metastatic carcinoma.

Accessory spleens, also known as supernumerary spleens, splenunculi, or splenules, are congenital foci of healthy splenic tissue that are separate from the main body of the spleen [1]. They arise from the failure of fusion of the 


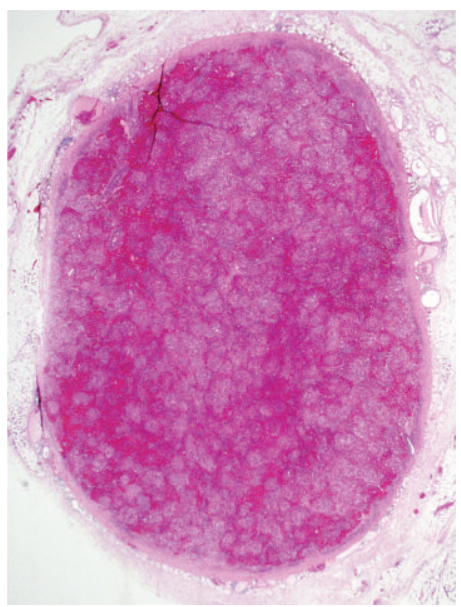

(a)

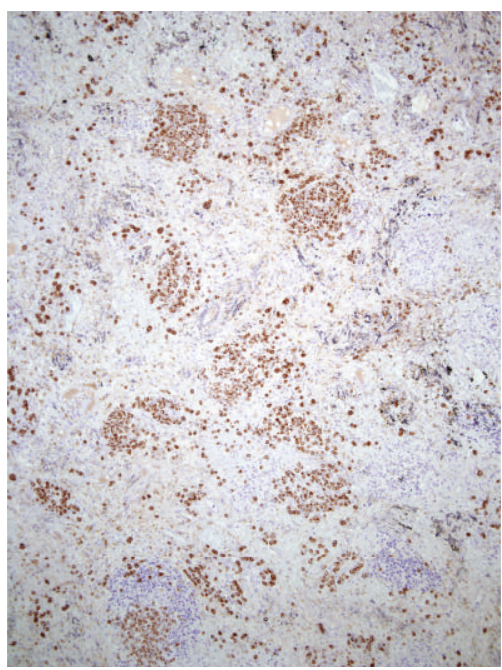

(d)

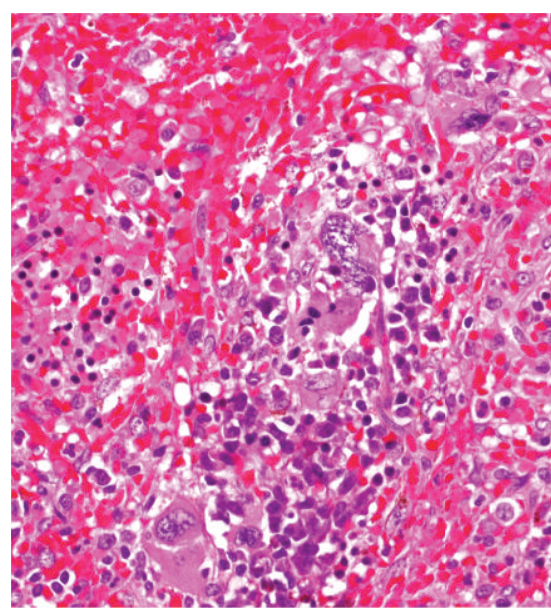

(b)

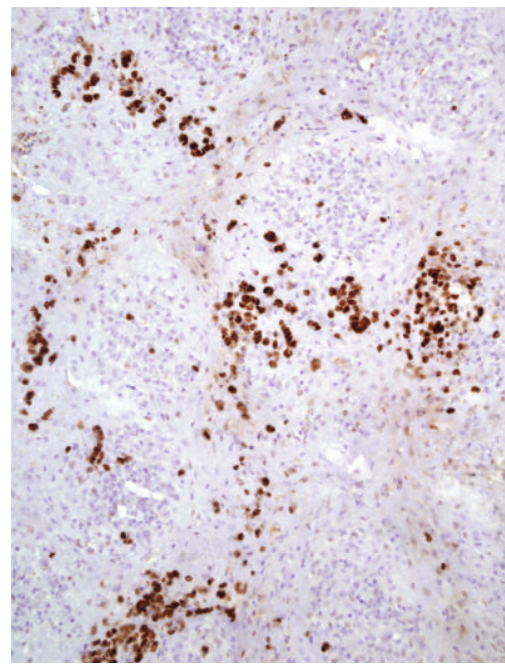

(e)

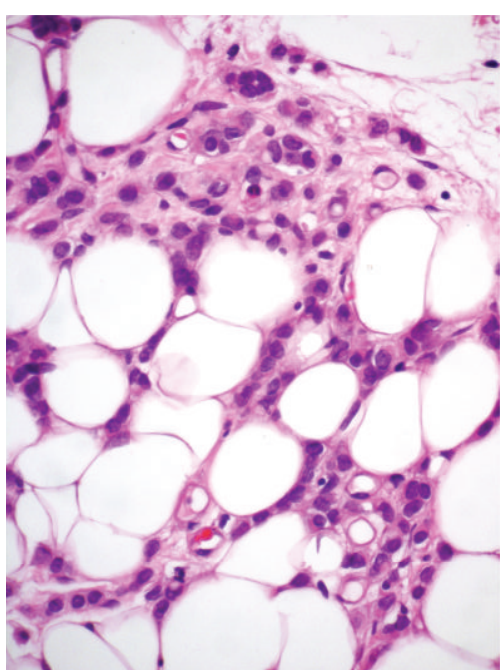

(c)

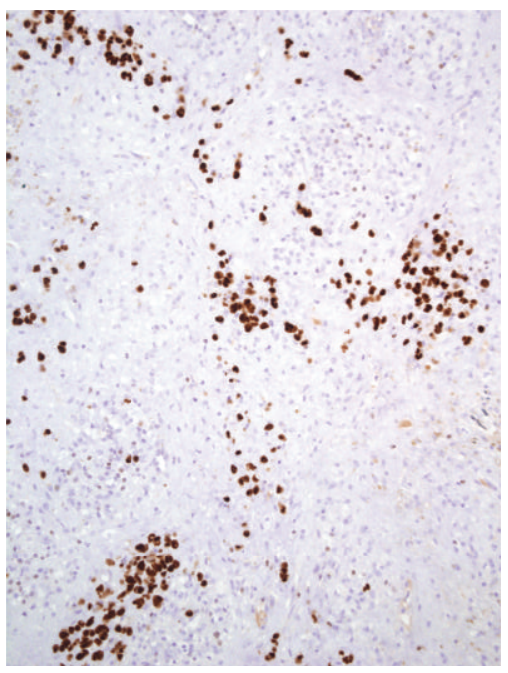

(f)

FiguRE 2: (a) Low power microscopic view of the accessory spleen showing marked congestion and a nodular pattern (hematoxylin and eosin stained section, magnification $\times 20$ ). (b) High power view shows extramedullary hematopoiesis including atypical megakaryocytes (hematoxylin and eosin stained section, magnification $\times 400$ ). (c) Small cells with occasional cytoplasmic vacuoles resembling lobular carcinoma cells infiltrate the fatty tissue (hematoxylin and eosin stained section, magnification $\times 400$ ). (d) Cytokeratin stain highlights numerous cells that represent metastatic carcinoma (magnification $\times 100$ ). (e) Estrogen receptors and (f) GATA3 strongly stain the nuclei of the cancer cells confirming the mammary origin of the cancer $((e)$ and (f) magnification $\times 200)$.

splenic anlage, located in the dorsal mesogastrium, during the fifth week of fetal life [2]. Accessory spleens are relatively common and are seen in $10-30 \%$ of patients at autopsy [13].

Metastases from solid tumors to accessory spleens are extremely rare. To our knowledge only two such cases were reported previously. Mihmanli et al. [5] described a case of ovarian carcinoma metastatic to a mass located in the transverse mesocolon which resulted to be an accessory spleen containing metastatic ovarian carcinoma. The second case, reported by Porwal et al. [6], was a retroperitoneal, suprarenal accessory spleen infiltrated by metastatic transitional cell carcinoma of the kidney.
Metastatic carcinoma of the spleen is an uncommon clinical problem [13]. Most splenic metastases develop in patients with multivisceral metastatic disease being skin melanoma and breast, lung, ovarian, colorectal, and gastric carcinomas the most common primary sources [14, 15]. Rarely, breast carcinoma metastatic to the spleen may present as solitary splenic metastatic masses [16-19] or associated with idiopathic (immune) thrombocytopenic purpura [20]. The relative paucity of splenic epithelial metastases may be a result of proapoptotic signals in the spleen, leading to decreased epithelial survival [21].

Metastatic lobular carcinoma of the breast, unlike the ductal type, can be difficult to recognize in routine histologic 
sections [11]. Bland nuclear features, a discohesive infiltration pattern, and the frequent lack of a desmoplastic stromal reaction contribute to this difficulty. Moreover, the occurrence of occult lymph node metastasis from breast cancer has been estimated to be about $20 \%$, with a rate as high as $39 \%$ being reported in lobular carcinoma [12]. Likewise, Lyda et al. [22] documented that keratin immunohistochemistry detected clinically significant bone marrow metastases of lobular breast carcinoma in which the initial histologic examination was negative.

In the present case both the spleen and accessory spleen displayed severe congestion, extramedullary hematopoiesis $(\mathrm{EMH})$, and marked nodular fibrosis. Carcinoma cells were not evident on hematoxylin and eosin stained sections and were only disclosed with cytokeratin stain. To confirm the mammary origin of the cancer cells we employed estrogen receptors and GATA3 immunostains. The latter is a novel transcription factor belonging to the GATA family, proved to be a useful immunohistochemical marker for several malignancies, mainly breast and urothelial carcinomas [23, 24]. Miettinen et al. [23] found that $92 \%$ and $96 \%$ of primary and metastatic mammary ductal carcinomas, respectively, and $100 \%$ of mammary lobular carcinoma were diffusely positive for this marker. However, as other tumors such as chromophobe renal cell carcinoma, basal cell carcinoma, and mesothelioma have been reported to be reactive to GATA3 [24], it is advisable to use more than one marker to confirm the mammary origin of a metastatic carcinoma.

In summary, to our knowledge this is the first report of breast carcinoma metastasizing to an accessory spleen. It underscores the importance of getting a comprehensive patient history and conducting immunohistochemical stains in relevant cases to avoid missing a diagnosis of metastatic carcinoma.

\section{Competing Interests}

The author declares that there is no conflict of interests regarding the publication of this paper.

\section{References}

[1] J. L. Freeman, S. Z. Jafri, J. L. Roberts, D. G. Mezwa, and A. Shirkhoda, "CT of congenital and acquired abnormalities of the spleen," RadioGraphics, vol. 13, no. 3, pp. 597-610, 1993.

[2] W. J. Dodds, A. J. Taylor, S. J. Erickson, E. T. Stewart, and T. L. Lawson, "Radiologic imaging of splenic anomalies," American Journal of Roentgenology, vol. 155, no. 4, pp. 805-810, 1990.

[3] G. Gayer, R. Zissin, S. Apter, E. Atar, O. Portnoy, and Y. Itzchak, "CT findings in congenital anomalies of the spleen," British Journal of Radiology, vol. 74, no. 884, pp. 767-772, 2001.

[4] B. Halpert and Z. A. Alden, "Accessory spleens in or at the tail of the pancreas. A survey of 2,700 additional necropsies," Archives of Pathology, vol. 77, no. 6, pp. 652-654, 1964.

[5] V. Mihmanli, G. Toprakci, N. Cetinkaya, A. Kilickaya, and G. Kamali, "Intraparenchymal metastasis to the accessory spleen from ovarian cancer: a case report," European Journal of Gynaecological Oncology, vol. 34, no. 6, pp. 580-581, 2013.
[6] R. Porwal, A. Singh, and P. Jain, "Retroperitoneal accessory spleen presented as metastatic suprarenal tumour- a diagnostic dilemma," Journal of Clinical and Diagnostic Research, vol. 9, no. 6, pp. PD07-PD08, 2015.

[7] M. W. Mulholland, K. D. Lillemoe, G. M. Doherty, R. V. Maier, D. M. Simeone, and G. R. Upchurch Jr., Greenfield's Surgery: Scientific Principles and Practice, vol. 74, Lippincott Williams \& Wilkins, Philadelphia, Pa, USA, 5th edition, 2011.

[8] S. R. Lakhani, E. Rakha, and P. T. Simpson, "Invasive lobular carcinoma," in WHO Classification of Tumours of the Breast, S. R. Lakhani, I. O. Ellis, S. J. Schnitt, P. H. Tan, and M. J. van de Vijver, Eds., pp. 40-42, IARC, 4th edition, 2012.

[9] S. Ferlicot, A. Vincent-Salomon, J. Médioni et al., "Wide metastatic spreading in infiltrating lobular carcinoma of the breast," European Journal of Cancer, vol. 40, no. 3, pp. 336-341, 2004.

[10] J. Lamovec and M. Bracko, "Metastatic pattern of infiltrating lobular carcinoma of the breast: an autopsy study," Journal of Surgical Oncology, vol. 48, no. 1, pp. 28-33, 1991.

[11] M. A. Bitter, D. Fiorito, M. E. Corkill et al., "Bone marrow involvement by lobular carcinoma of the breast cannot be identified reliably by routine histological examination alone," Human Pathology, vol. 25, no. 8, pp. 781-788, 1994.

[12] R. J. Cote, H. F. Peterson, B. Chaiwun et al., "Role of immunohistochemical detection of lymph-node metastases in management of breast cancer," The Lancet, vol. 354, no. 9182, pp. 896-900, 1999.

[13] E. Compérat, A. Bardier-Dupas, P. Camparo, F. Capron, and F. Charlotte, "Splenic metastases: clinicopathologic presentation, differential diagnosis, and pathogenesis," Archives of Pathology and Laboratory Medicine, vol. 131, no. 6, pp. 965-969, 2007.

[14] T. Berge, "Splenic metastases: frequencies and patterns," Acta Pathologica and Microbiologica Scandinavica, vol. 82, no. 4, pp. 499-506, 1974.

[15] K. Y. Lam and V. Tang, "Metastatic tumors to the spleen: a 25-year clinicopathologic study," Archives of Pathology and Laboratory Medicine, vol. 124, no. 4, pp. 526-530, 2000.

[16] M. Barreca, D. Angelini, A. Gallo, F. Puntillo, P. M. Amodio, and E. Fernandes, "Single asymptomatic splenic metastasis of breast carcinoma: report of a clinical case," Il Giornale di Chirurgia, vol. 22, no. 6-7, pp. 227-228, 2001.

[17] S. Iype, M. A. Akbar, and G. Krishna, "Isolated splenic metastasis from carcinoma of the breast," Postgraduate Medical Journal, vol. 78, no. 917, pp. 173-174, 2002.

[18] F. J. Hoar, S.-Y. Chan, P. S. Stonelake, R. W. Wolverson, and D. Bareford, "Splenic rupture as a consequence of dual malignant pathology: a case report," Journal of Clinical Pathology, vol. 56, no. 9, pp. 709-710, 2003.

[19] K. Pailoor, H. Fernandes, and C. R. S. D’Souza, "Rare case of isolated splenic metastasis from the breast," Basic and Applied Pathology, vol. 5, no. 3, pp. 76-78, 2012.

[20] A. M. Peters, "Why the spleen is a very rare site for metastases from epithelial cancers," Medical Hypotheses, vol. 78, no. 1, pp. 26-28, 2012.

[21] O. W. Cummings and M. T. Mazur, "Breast carcinoma diffusely metastatic to the spleen: a report of two cases presenting as idiopathic thrombocytopenic purpura," American Journal of Clinical Pathology, vol. 97, no. 4, pp. 484-489, 1992.

[22] M. H. Lyda, M. Tetef, N. H. Carter, D. Ikle, L. M. Weiss, and D. A. Arber, "Keratin immunohistochemistry detects clinically significant metastases in bone marrow biopsy specimens in 
women with lobular breast carcinoma," The American Journal of Surgical Pathology, vol. 24, no. 12, pp. 1593-1599, 2000.

[23] M. Miettinen, P. A. McCue, M. Sarlomo-Rikala et al., "GATA3: a multispecific but potentially useful marker in surgical pathology: a systematic analysis of 2500 epithelial and nonepithelial tumors," American Journal of Surgical Pathology, vol. 38, no. 1, pp. 13-22, 2014.

[24] N. G. Ordoñez, "Value of GATA3 immunostaining in tumor diagnosis: a review," Advances in Anatomic Pathology, vol. 20, no. 5, pp. 352-360, 2013. 


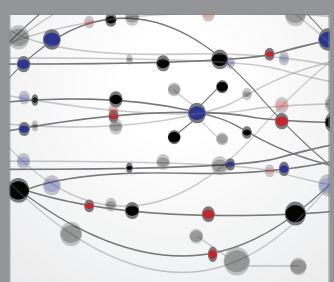

The Scientific World Journal
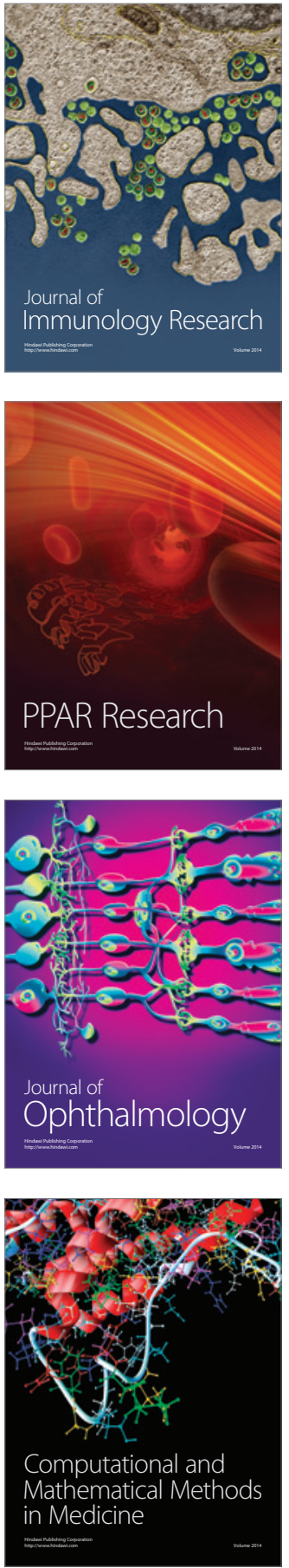

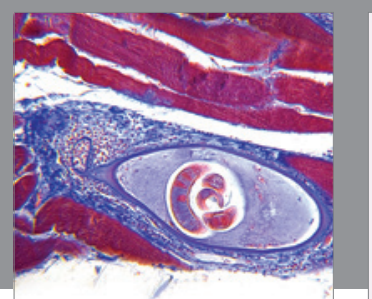

Gastroenterology Research and Practice

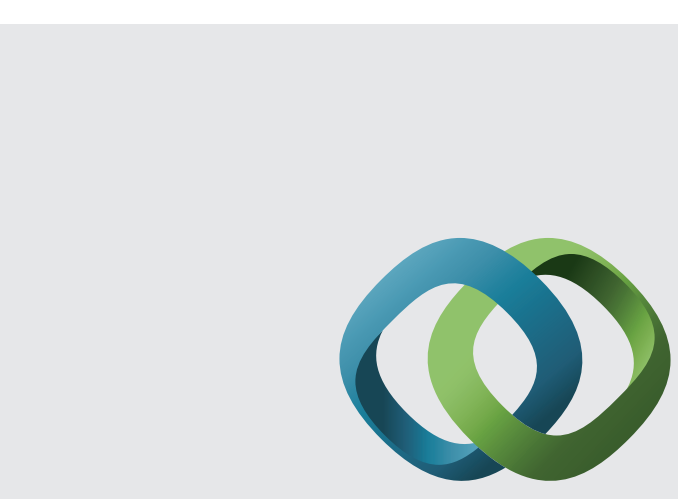

\section{Hindawi}

Submit your manuscripts at

http://www.hindawi.com
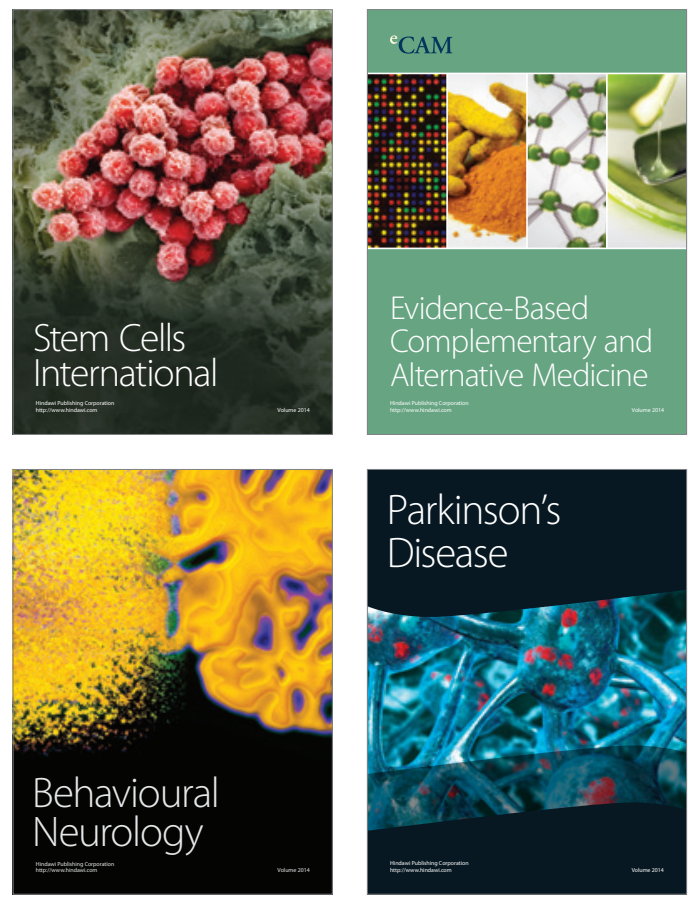
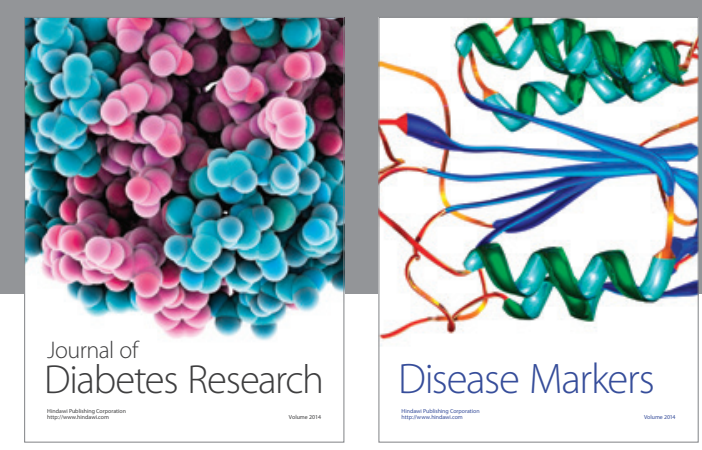

Disease Markers
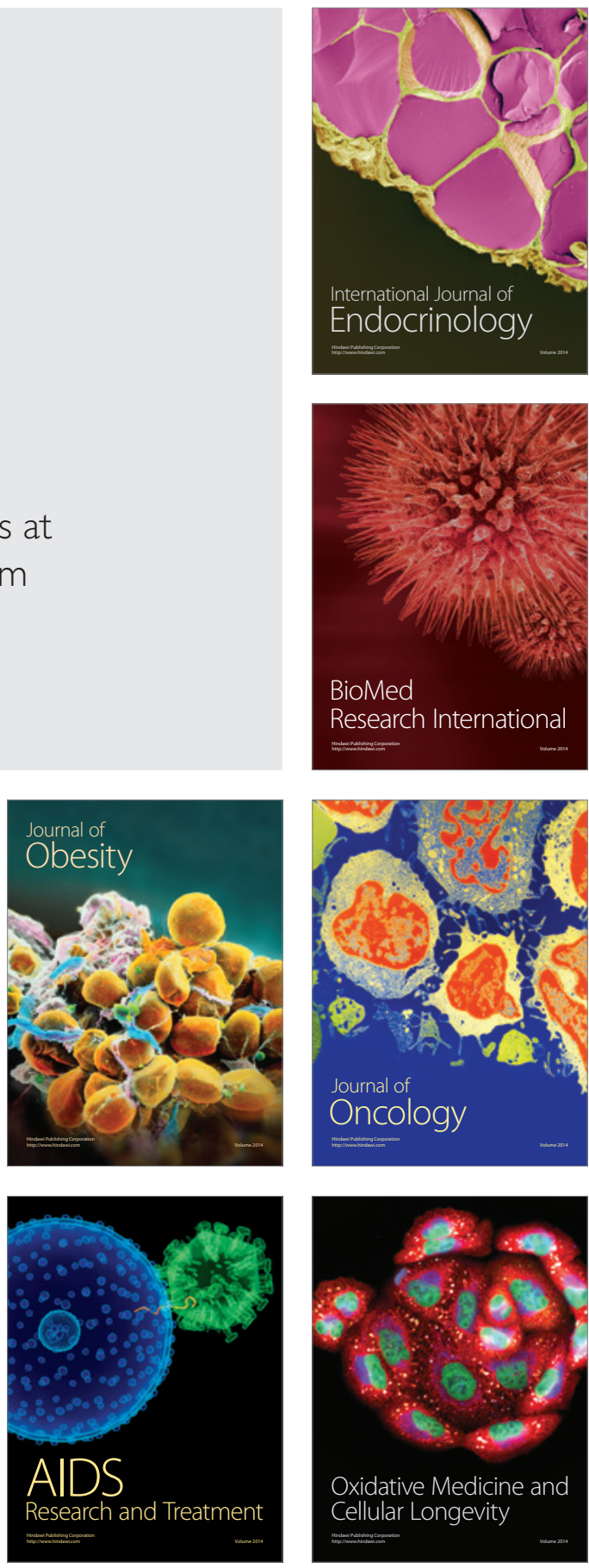